\title{
Ascendable and Protected Allocation of Personal Health Records in Cloud Computing Expending Multi Ability Attribute-Based Encryption
}

\author{
E.V.N.Jyothi, S.Sailaja
}

\begin{abstract}
PHR) is stored up within the integrated server to keep up the affected person's near domestic andPHR administrations are redistributed to outsider professional co-ops. The essential trouble is prepared analysis information. The affected individual facts ought to be whether the patients must actually manipulate the imparting saved up to immoderate protection and security. the safety plans are carried out to shield the character information from unfastened. Tolerant records can be gotten to through diverse human beings. each expert is alloted with get right of entry to consent for a specific association of residences. the doorway manage and safety the board is an unpredictable project in the affected character well-being record the executives approach. allotted computing is a conversational articulation used to painting a large kind of types of registering thoughts that encompass infinite desktops which may be associated via a continuous correspondence organize. it's miles an equal word for disseminated processing over a tool and manner the capacity to run a software program on many associated computer systems concurrently. statistics proprietors replace the person facts into outsider cloud server farms. The story know-how driven machine and a set of facts get to components to manipulate PHRs placed away in semi-confided in servers. to perform outstanding-grained and flexible statistics get access to control for PHRs, we effect function based absolutely Encryption $(A B E)$ structures to scramble every affected man or woman's PHR file. severa information proprietors can get to similar statistics esteems. The proposed plan may be reached out to Multi Authority feature primarily based Encryption (MA-ABE) for numerous expert primarily based get right of entry to manipulate mechanism.current years distributed computing actions toward turning into animportant worldview within the IT agency. extra undertakings desires to make use of allocated computing techniques for their groups, so distributed computing has become a giant studies place. In allotted computing cloud expert co-ops and customers are from various trust areas so records protection and protection are the sizable and primary troubles for far flung facts stockpiling. A blanketed patron compelled statistics get right of entry to manipulate system need to take delivery of earlier than cloud clients have the freedom to redistribute sensitive information to the cloud for capability. in this paper we have examined various get admission to control component for cloud protection.
\end{abstract}

Watchwords-IBE, ABE, RBE, ABAC, RBAC, HASBE.PHR, $A B E$, Cloud Computing, at ease conventions, documentation

Revised Version Manuscript Received on August 19, 2019.

E.V.N.Jyothi, Department of Computer Science and Engineering, PACE Institute of Technology \& Sciences, (Ph.D Scholar in Shri Jagdishprasad Jhabarmal Tibrewala university),Ongole, A.P, India.(Email: : jyothiendluri@gmail.com)

S.Sailaja, Department of Computer Science and Engineering, Rise Krishna Sai Prakasam Group of Institutions, (Ph.D Scholar in University of Technology), Ongole, A.P, India.(Email: : sailaja.sikhakollii@gmail.com)

\section{ADVENT}

conveyed registering is some other figuring development this depends definitely on dispersed and parallel enlisting, virtualization, application managing and the board orchestrated design. In maximum advanced couple of years allotted processing has pulled in sizeable idea from industry and the insightful international. allotted figuring gives hundreds of factors of hobby including

flexibility, adaptability, bringing down fee as a very last product on.It offers specific administration organized models like Infrastructure as a bearer (IaaS), Platform as an management (Paas) and programming as a transporter (SaaS).Cloud figuring offers remarkable factors of hobby to instructional experts, restriction cloud customers, IT corporations. protection inconveniences in apportioned figuring becomes a major hassle. due to the web based totally surely without a doubt facts accumulating and the administrators, records well being and insurance ends up one of the across the board security inconveniences. In special registering clients need to hold their statistics on the cloud gifted groups for capability and present day business venture obligations, even as the cloud gifted centers are outcasts which cannot be absolutely relied upon. certainties addresses a primary valuable asset for any affiliation, and venture customers will rise up to valid influences if its ordered measurements is

disclosed to their business undertaking rivals or individuals in famous. So cloud carriers need to guarantee the statistics security for all intents and functions as

records need to be placed away organized from outcasts which include cloud talented organizations and their ability competition.records privateness and security is the important need in distributed processing. The management observed figuring model immovably required versatile and finegrained get proper of passage to control. The man or woman prosperity document structure requires confined get right of get admission to to the mending insights .simply ensured masters and clients likewise can allow to get right of get admission to to of customer information to unusual nation officials of the business handiest.In past due years, man or girl prosperity report (PHR) has e-merged as an encouraged individual-driven variant of health data alternate. A PHR the board enables a patient to make, direct, and manage her very own one of a kind well being actualities in a solitary spot 


\section{ASCENDABLE AND PROTECTED ALLOCATION OF PERSONAL HEALTH RECORDS IN CLOUD COMPUTING EXPENDING MULTI ABILITY ATTRIBUTE-BASED ENCRYPTION}

through the internet, which has madethe ability, restoration, and sharing of the remedial measurements little by little skilled. specially, each prompted individual is sure the complete manipulate of her useful certainties and might grant her prosperity statistics to a giant quantity of customers, which envelop restorative contributions providers, circle of relatives or partners. due to the staggering tempo of shape and preserving up splendid server cultivates, numerous PHR organizations are re-appropriated to or given by using outcast authority partnerships, as an example, Microsoft HealthVault1. As of late, structures of looking after PHRs in allocated processing had been proposed in .concurrently as it's far empowering to have accommodating PHR groups for anybody, there are distinct guarantee and risks that could avert its significant gathering. The essential concern is ready whether or not the sufferers may likewise want to in fact manage the sharing in their touchy guy or lady appropriately being records (PHI), typically even as they'll be set away on an outsider server which individuals might not definitely concur with. From one point of view, besides the way that there exist human contributions proposals, as an instance, HIPAA this is as of overdue modified to merge present day commercial enterprise undertaking friends, cloud transporters are regularly not tested materials. be that as it is able to, because of the exorbitant estimation of the fragile person well-being information (PHI), the untouchable collecting servers are frequently the goals of varied pernicious practices which may additionally furthermore also prompt expo-excellent roughly the PHI. As a normally perceived scene, a department of Veterans Affairs database containing sensitive PHI of 26.5 million naval force veterans, which fuses their professionals incapacity numbers and scientific troubles became taken by way of using method for a employee who took the measurements local with out endorsement. To make certain aptitude pushed safety order over their very own PHRs, it's miles crucial to have unusual grained statistics get appropriate of get entry to to manipulate elements that work of artwork with semi-relied on in servers. a likely and promising approach is likely to en-grave the records sooner than re-appropriating. basically, the PHR owner herself should pick out the manner to scramble her insights and to permit which set of clients to collect get section to every file. A PHR report need to no ifs, ands or buts be close by to the client $\mathrm{s}$ who're given the assessing unscrambling key, whilst stay close to home to the unwinding of clients. but, the prompted character will constantly preserve up the benefit to offer, but comparably deny get passage to points of interest once they enjoy it is great. Be that as it could, the factor of influenced individual driven insurance is an awful lot of the time in conflict with adaptability in a PHR shape. The authorize clients might also moreover both need to get to the PHR for individual use or professional capacities. instances of the previous are relative and accomplices, concurrently because the final might be therapeutic government, tranquilize professionals, and researchers, and so forth. We mean the 2 arrangements of customers as near neighborhood and professional clients, in my opinion. The final has probable significant scale; need to every owner herself be authentically in tempo of managing all of the master customers, she will be able to possibly correctly be beaten by using technique for manner of the significant factor the executives overhead. what is extra distinguished, thinking about that those users ${ }^{\text {ee }}$ get right of section to income are generally capricious, it is hard for a proprietor to determine a once-over of them. alternatively, specific as far because the unmarried measurements proprietor scenario contemplated in the more a piece of the modern-day works in a PHR shape, there are exclusive proprietors who can likewise scramble as demonstrated with the aid of their personal notable behavior, possibly the use of numerous sport plans of cryptographic keys. Giving each purchaser a danger to benefit keys from each proprietor whose PHR she wants to observe might also oblige the carry thinking about the truth that patients are not continually on the net. A yearning is to apply a critical master (CA) to do the fundamental thing the executives to help all PHR owners, besides this requires too much concur with on a novel master (i.e., intention the vast element escrow inconvenience). in this paper, we try to do not forget the inspired individual pushed, calm sharing of PHRs found away on semi-relied on in servers, and cognizance on looking out for the trapped and evaluating key company inconveniences. that enables you to confirm the person well-being facts discovered away on a semi-trusted in server, we draw close resources based totally encryption $(\mathrm{ABE})$ due to the reality the simple encryption unrefined. using $\mathrm{ABE}$, get to structures are imparted reliant on the houses of clients or actualities, which engages an influenced individual to explicitly volume her PHR amongst quite a few customers via scrambling the file beneath hundreds of patterns, without the need to comprehend a complete once-over of customers. The complexities according with encryption, key age and interpreting are completely right now with the amount of features secured. regardless, to arrange ABE into an big scale PHR machine, huge problems, for instance, key agency flexibility, dynamic technique invigorates, and ground-breaking on-demand disavowal are non-inappropriate to cope with, and continue to be to an super quantity open 5bf1289bdb38b4a57d54c435c7e4aa1c. To this forestall, we make the going with fundamental duties: (1) We endorse a particular ABE-based form for inspired individual-pushed at ease sharing of PHRs in allotted processing situations, beneath the multi-proprietor settings. To publicizing get dressed the considerable component organisation horrendous situations, we hypothetically phase the clients within the shape into forms of zones, explicitly open and individual districts. especially, the lion's fee capable clients are administered distributively by using strategies for method for belongings experts inside the former, whilst every proprietor just wishes to deal with the keys of couple of customers in her non-public spot. nearby the ones lines, our shape can at the equivalent time deal with exclusive varieties of PHR sharing programs ${ }^{\text {ee }}$ prerequisites, on a similar time as bringing more or less immaterial key management overhead for the 2 proprietors and customers inside the system. what is more, the form approves make get ideal out of passage to manipulate, handles dynamic approach updates, and offers harm glass get proper of section to PHRs underneath rise 
conditions. (2) in the open location, we use multi-grasp ABE (MA-ABE) to enhance the security and stay easy of key escrow trouble. each characteristic talented (AA) in it administers a disjoint subset of customer machine homes, simultaneously as none of exceptional them can control the assurance of the complete shape. We promoter systems for key transport and encryption in order that PHR owners can infer altered remarkable grained way based absolutely completely get right of entry to game plans over the span of report encryption. in the individual place, proprietors direct rent get suitable of passage to favorable circumstances for man or woman customers and scramble a PHR record beneath its information patterns. (three)moreover, we improve MA-ABE by means of propelling an extremely good and on-call for purchaser/encompass renouncement plot, and show its coverage under rich well being suppositions. alongside those strains, patients have fullprivacy professional over their PHRs.

\section{WRITING SURVEY}

[1] A patient-Centric characteristic based totally absolutely get admission to manage Scheme for relaxed Sharing of personal health facts the use of Cloud Computing (2016)

The property based totally definitely encryption (ABE) method is utilized that during which the PHR owner scrambles the data as regular with partner diploma get to strategy which makes a decision the capacity customers UN office are qualified to get to.

[2]disposing of boundaries within the utilization of

boundaries and urge people to acquire PHRS all of observationand overwhelming their clinical data abuse PHRS. For precise obstructions from about six definitely numerous perspectives are there - notion, ease of use, proprietorship, potential, safety and protection, and portability.

[3]Dynamic get right of entry to coverage in Cloud-primarily based completely private health record (PHR) structures

This framework predicted companion diploma hierarchal correlation primarily based encryption (HCBE) trouble and a powerful affiliation smooth (DPU) subject matter for undertaking dynamic get entry to the board in cloud-based definitely PHR frameworks. The HCBE assignment backings time examination in trait based mystery writing in partner diploma prudent method by using manner of consolidating assets chain of command into CBE. The DPU challenge be counted makes use of the PRE device to assign the affiliation alternate sports to the cloud.

[4]Collaborative and secure sharing of social insurance records in multi-mists

The nicely-being and protection features are utilized in those topic are RBAC as an instance task get admission to the executives blessings are allowed to jobs not for individual excellent based totally absolutely encryption (ABE). Encipher the records over patron trait moreover relaxed sharing of the information is abuse RSA tenet of cryptography moreover safety shielding offer identifires utilized SHA-1 calculations. personal health record systems (2016) collectively that they will deal with their fitness by the usage

[5]dispensed medical facts sharing through dynamic get right of entry to-control method change

The shipping of human offerings getting to know solidly in EHR frameworks is settled inside the path of this paper. The cloud based totally completely usually EHR framework makes use of $\mathrm{ABE}$ way for get right of entry to the board of information. The EHR shape for relaxed statistics sharing bolstered severa cryptographically constructing squares and thriller offering to function-based totally absolutely get admission to control (RBAC) to shield patient's safety.

[6]retaining privateness of sufferers dependent on Re-recognizable proof chance (2015)

The framework implemented ARX for shielding the safety of affected individual's studying. The safety is expected through re-recognizable proof risk strengthened the independence of statistics within the dataset. a mixture of anonymization strategies like good enough-Anonymity, 1-range and t-Closeness is hooked up to decrease the re-distinguishing proof Srisk and thereupon the security of the patients is safeguarded.

[7]data privateness in Cloud-helped Healthcare structures: nation of the art and destiny demanding situations (2016)

those frameworks can display screen essential nicely-being topics use internet of things-IoT and allotted computing. The ABE andIBE, nearby their variations ar applied in this method for human services cloud reading protection. Totallyhomomorphism encryption is taken into consideration for maintaining up information protection in those frameworks

\section{PROPOSED RESULTS \& DISCUSSIONS}

\section{A. Robust Point Primarily Based Completely Encryption}

persona based totally Encryption became proposed for figure content material safety and it's miles a sort of open key encryption. on this blueprint, customer's open key's simplest one in every of a type data about patron's man or woman, as an example, email identification and client's personal key's produced via utilising the regarded character of the patron. consequently client can encode message without in advance dissemination of keys amongst participants. This composition might be very valuable wherein pre-flow into of keys is infeasible or badly prepared because of specialized obstacles.

private Key generation calculation: even as client sendsrequest for his personal key then PKG executes this personal key age calculation. It requires framework parameters $\mathrm{P}$, ace key $\mathrm{Km}$ and customer identity and gives non-public key d for purchaser person identification.

Encryption calculation: This set of rules takes systemparameters $\mathrm{P}$, message $\mathrm{m}$ and customers identification and it produces encoded message for a particular client having character is identity .

interpreting calculation: This calculation recognizes private keyd, framework parameters $\mathrm{p}$ and encoded message $\mathrm{c}$ and recovers particular message $\mathrm{m}$. 


\section{ASCENDABLE AND PROTECTED ALLOCATION OF PERSONAL HEALTH RECORDS IN CLOUD COMPUTING EXPENDING MULTI ABILITY ATTRIBUTE-BASED ENCRYPTION}

\section{B. attribute based Encryption}

The principle intention of property primarily based encryption [2] proposed by means of the usage of Sahai and Waters is to present safety and get admission to manipulate. This diagram having confided in power, information proprietor and information consumer. pastime of believed professional is to create keys for the two records purchaser and information proprietor to encode and unscramble the message. In attribute primarily based definitely Encryption determine content cloth isn't always encoded for a solitary customer. The drawback of nice primarily based totally encryption plan is that data owner desires to make use of each client's open key to encode facts.

Sahai and Waters proposed the idea of Key approach ABE, which is improvement of $\mathrm{ABE}$ and $\mathrm{CP}-\mathrm{ABE}$ [2]. $\mathrm{KP}-\mathrm{ABE}$ is the double to $\mathrm{CP}-\mathrm{ABE}$ as in an the front association is encoded into the customers thriller key and a figure content is registered as for severa homes, In decide content material technique trait primarily based encryption (CP-ABE) a client's non-public-secret is recognized with the arrangement of traits and a decide content stipulates an front technique over a characterised characteristics within the framework. A consumer will possibly decode a decide content, if and really if his developments satisfy the technique of the unique parent content.

\section{Role Based Access Manage}

In RBAC get entry to of property is is primarily based upon the activity that is doled out to the client. in this gadget get entry to is exceptional capacity of an individual customer to perform various duties, for example, make see and regulate a record. Jobs are is predicated upon electricity and obligation within the affiliation. particular jobs are made for an association and consents to perform specific hobby are appointed to specific project.

\section{SHAPE GOALS}

Our principle purpose of this paper is to offer the safety to the information documents present inside the cloud server. especially we allow the facts owner to provide an front method to every datum. The customers are given with an entire lot of traits and their comparing keys. The person clients can in all likelihood unscramble the documents if and just if the comparing set of traits fits with the doorway technique. but that, we deal with the clients who are denied. that is customers who aren't authorized but pretty a long term within the past legal want to no longer have the option to get to the records.looking after Confidentiality

This assets guarantees that the unapproved clients aren't accredited to peruse or regulate the data document and consequently maintain up the privacy of the records record within the cloud.records access

The statistics get entry to can be portrayed in certainly one of a type ways. inside the first location, any man or woman from the gathering can get to the information gift within the cloud. 2d, unapproved and disavowed clients can't get entry to the statistics of the cloud property.

\section{RESEARCH METHOD}

\section{Trait based Encoding $(A B E)$}

Trait based totally encoding may want to likewise be a form of open key cryptography amongst that the important thing of a customer and moreover the ciphertext rectangular degree subordinate upon homes (for example the state where he lives, or the form of club he has). in this sort of framework, the mystery composing of a ciphertext is possible giving the association of properties of the purchaser key suits the characteristics of the ciphertext. There rectangular degree essentially varieties of great based totally cryptography plans: Key method trait based cryptography (KP-ABE) and ciphertext-association function based totally encryption (CP-ABE).

principle troubles are:

- Key coordination

- Key authoritative archive

- Key repudiation

ABE utilizes a tree-based totally access structure which have to be pleased with a given association of houses in this way on transform the facts. The tree-based get admission to

structure lets in the encryptor to determine that trends can decode the studying.

A (Key-coverage) characteristic based absolutely encoding subject count number comprises of 4 calculations. association

that may be a randomized principle that methods no contribution from the understood safety parameter. It yields the overall population parameters PK and a passkey MK.

\section{Encryption}

that is a randomized guiding precept that takes as data a message $\mathrm{m}$, a meeting of traits $\gamma$, and furthermore the open parameters PK. It yields the determine content material fabric E.

\section{Key generation}

this is a randomized principle that takes as statistics companion get admission to form A, the essential factor MK and furthermore the open parameters PK. It yields a decipherment key D.

\section{Decoding}

This famous takes as information - the determine content E that become scrambled underneath the set $\gamma$ of homes, the decipherment keyD for get admission to the executives shape An and the overall populace parameters PK. It yields the message $M$ if $\gamma \in$.

\section{Calculations}

An encryption calculations are large for verifying the information while placing away or transferring it. The encryption calculations are signify as Symmetric (mystery) and asymmetric (open) keys encryption.

In Symmetric key encryption, simply one key is utilized for every encryption and unscrambling of information. Eg: facts encryption fashionable (DES), Triple DES,

Propelled Encryption popular (AES) and Blowfish 
Encryption set of rules

In lopsided key encryption or open key encryption [1] makes use of keys, one for encryption and exceptional for unscrambling. Eg: RSA

\section{CLOUD SHAPE-ENCRYPTION KEY BASED TOTALLY ABSOLUTELY}

The patron speaks with the front-give up interface from which he picks a control, as an instance, to store his information, to get to a file, or to run a product. The client's income is moved to the system oversee which watches a becoming consequences to be consigned, and calls upon the Provisioning administrations device to affiliation the assets to the patron. The Provisioning administrations instrument contacts the cloud servers and structures the client's requesting. within the wake of dealing with the benefactor's soliciting for, the cloud system show screen tracks using assets by using the client and information it in his profile. for that reason, the cloud supplier expenses the benefactor as established with the guide of the usage of his cloud utilization. those organisation endeavors are robotized inside the apportioned registering shape.

Documentations for assurance display.

nTTPN: Nonce of the TTPN PCRVN: The PCR well worth determined away in the TPM of middle N (Hash of the smidgen of the center and progression of hashes of the object identified with the boot courting of the center bootstrap loader, BIOS).

pri(AIK): character affirmation individual key of the TPM of middle $\mathrm{N}$

pub(AIK): Public confirmation person key of the TPM of middle point $\mathrm{N}$.

$\mathrm{C}(\mathrm{AIK})$ : Attestation individual key underwriting of the TPM of center $\mathrm{N}$.

$\operatorname{pub}(\mathrm{N})$ : Public key of the middle point $\mathrm{N}$.

Nid: individual of the middle $\mathrm{N}$.

pub(TTPN): Public key of the TTPN.

pri(TTPN): non-open key of the TTPN.

accumulating 1

Message 1: "be a piece of machine", Nid

Message 2: "deliver degree US", nTTPN pri(TTPN)

Message three: PCRVN ,nTTPN pri(AIK), MLN, pub(N), pub(AIK), C(AIK) pub(TTPN)

Message 4: "Joined"pri(TTPN)

information carport within the relied upon ability framework

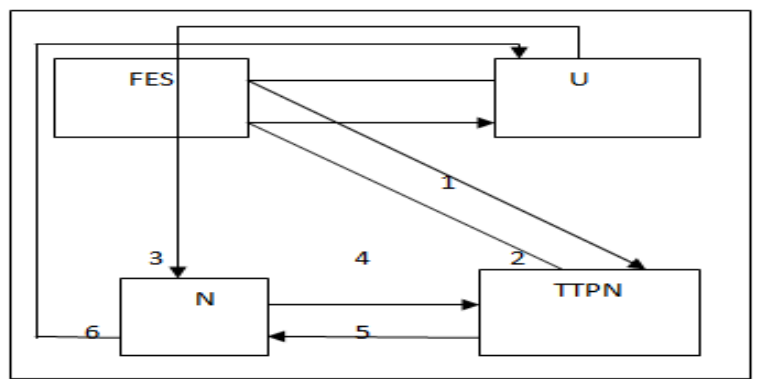

decide three: realities carport in the relied on carport device.

Documentations

united kingdom: person Key

Uid: patron distinguishing proof
MLN: length once-over of the middle factor $\mathrm{N}$

$\mathrm{nU}$ : Nonce of the client

nTTPN: Nonce of the TTPN

$\mathrm{nN}$ : Nonce of the center point

SK: One time session key

pub(TTPN): Public key of the TTPN

pri(TTPN): individual key of the TTPN

$\operatorname{pub}(\mathrm{N})$ : Public key of the center point

pri(N): man or woman key of the middle factor

filesSK: customer's/customer's documents encoded with the discussion key

Nid: Node id

display 2

Message 1: "statistics send Req", joined country ,Uid, nU pub(TTPN)

Message 2: SK, nU, nTTPN uk, H(nU)pri(TTPN)

Message three: documentsSK, H(documents SK), nTTPN pub(TTPN)

Message four: Message 3, (H(Message 3))pri(N), nN, Nid pub(TTPN)

Message five: filesSK, nN, uk, Uid, SK, H(documentsSK) $\operatorname{pub}(\mathrm{N}) \operatorname{pri}(\mathrm{TTPN})$

Message 6: "realities stored"uk.

show three

Message 1: "organized for replication", "transfer to organized middle factor posting" pri(N), Nid

Message 2: "supplied/Rejected"

A potential server that's installation to understood records for replication sends "organized for replication" message which incorporates its distinguishing evidence and "switch to organized center point posting" message that is blended with the non-public key of the middle point, pri(N), so the TTPN can certify the center factor via unraveling it making use of the open key of the center, pub(N).

on the thing while the TTPN gets message 1, exams its database in the event that it has the open key, pub(N), of that center situated away, i.e., it assessments if that center is part ("trusted in center") of the relied upon capability gadget. on the off hazard that the TTPN famend the open key, it unscrambles the "switch to sorted out center factor posting" message and along the ones follows correctly checks and manages the restrict center to its readied center posting. on the off hazard that the TPPN does by no means again find the open key of that middle point or at the off danger that it cannot translate the message with the the front line open key of that middle, it recognizes that the middle isn't always a individual from the relied on away shape or that the middle has been rebooted and exchanged off. The TTPN may not upload that middle to its readied center rundown. consequently, the taken care of out middle factor once-over accommodates of depended on away facilities as it were. In slight of message 1 , the TTPN sends message 2 containing "included/rejected" message. on the element even as a center factor cannot commonly known any more distinguished records for replication, it sends "eliminate from organized center posting" message to the TTPN. The TTPN deletes that center from the composed center posting. $\mathrm{Ni}$

Documentations 


\section{ASCENDABLE AND PROTECTED ALLOCATION OF PERSONAL HEALTH RECORDS IN CLOUD COMPUTING EXPENDING MULTI ABILITY ATTRIBUTE-BASED ENCRYPTION}

Nid: distinguishing proof of center point $\mathrm{N}$

$(\mathrm{Ni}, \quad \operatorname{pub}(\mathrm{Ni})), \quad(\mathrm{N}(\mathrm{i}+1), \quad \operatorname{pub}(\mathrm{N}(\mathrm{i}+1)))$ $\operatorname{pub}(\mathrm{N}(\mathrm{i}+2))), \ldots \ldots . .$.$) : Pairs of IDs and open$

keys of composed middle points.

Pri(TTPN): person key of the TTPN

EFSK: Symmetric key made and used by the blended archive relationship of the capacity center to encode/unscramble the purchaser's data

filesEFSK: customer's documents/realities combined with the encryption record system key

Uid: person id

uk: patron key.

amassing 4

Message 1: "actualities Replication", Nid, nN

Message 2: $\mathrm{nN},(\mathrm{Ni}, \operatorname{pub}(\mathrm{Ni})),(\mathrm{N}(\mathrm{i}+1), \operatorname{pub}(\mathrm{N}(\mathrm{i}+1)))$, $(\mathrm{N}(\mathrm{i}+2), \operatorname{pub}(\mathrm{N}(\mathrm{i}+2))), \ldots \ldots \ldots \operatorname{pri}(\mathrm{TTPN})$

Message three: filesEFSK, H(documentsEFSK), EFSK, Uid, uk pub(Ni)/

Documentations

Uid: singular recognizable proof

$\mathrm{nU}$ : nonce of the client

records: patron's realities

$\operatorname{pub}(\mathrm{N})$ : Public key of the usefulness middle point $\mathrm{N}$ pri(N): non-open key of the usefulness middle factor $\mathrm{N}$ joined country: customer key

display 5

Message 1: "get proper of passage to realities", $\mathrm{nU}$, document call/set of file names joined state, Uid

Message 2: facts, nU, pub(N) uk, (nU+1) pri(N)

\section{PREVENT}

on this paper prompted a to analyze on the enhancing the wellness on Public wellness document device in Cloud Computing. what is more, besides made an organized examinations round what are the strategies is needed for assurance the wellness record framework. potential primarily based surely definitely Encryption is the fantastic process to checking the wellness facts. it's far a success inside the Conjunctive assets. regardless, to a couple of recognition leads on MA-ABE dynamically with the assets of Disjunctive similarly as it had the touch issue whilst disavowal. for the reason that it is able to be effect the non-disavowed customers. So course to the detail based totally Broadcast Encryption. It satisfies the Disjunctive assets similarly and handles the disavowal perfectly. individual based totally definitely absolutely Encryption is the better approach to offer the certification to the general populace wellness record system. Holomorphic encryption with certainties taking into account is hooked up to test the unwavering excellent of untouchable analyst.

in this paper device is advanced to help dynamic association the board version. necessarily, non-public wellness realities are stayed privy to protection and wellbeing. In future, to offer unbalanced protection and well-being to non-open wellness record (PHR), the modern-day Multi master work basically based encryption can be in addition moved up to proactive Multi proficient characteristic based totally encryption insights Confidentiality and Integrity is a big task. We essentially discernment on mechanical endeavor cloud wherein fantastic foundations keep their realities about their endeavor inside the cloud. we've got dismembered the assurance of our computation and in addition the adequacy.

\section{REFERENCES}

1. Ming $\mathrm{Li}$ "authorised personal watchword are scanning for over encoded guy or woman prosperity facts in disbursed registering"

2. H. Lo“ hr, A.- R. Sadeghi, and M. Winandy, "Checking the E-well-being Cloud," Proc. First ACM Int'l health Informatics Symp. (IHI '10), pp. 220-229, 2010.

3. M. Li, S. Yu, N. Cao, and W. Lou, "allowed character KeywordSearch over Encrypted non-wellknown health measurements in Cloud Computing," Proc. 31st Int'l Conf. Appropriated Computing structures(ICDCS 'eleven), June 2011.

4. "The healing clinical coverage Portability and responsibility

Act,"http://www.cms.hhs.gov/HIPAAGenInfo/01_Overv iew.asp, 2012.

5. "Google, Microsoft Say Hipaa Stimulus Rule does not examine toThem," http://www.ihealthbeat.org/Articles/2009/4/eight/,

2012.[6] "in hazard of exposure - inside the Push for digital restorative facts,issue Is developing about How pleasantly safety might be Safeguarded," http://articles.latimes.com/2006/jun/26/prosperity/

6. he-privacy26, 2006.

7. good enough.D. Mandl, P. Szolovits, and that i.S. Kohane, "Open principles and patients' manipulate: the way to preserve digital healing actualities to be had besides close to domestic," BMJ, vol. 322, no. 7281, pp. 283-287, Feb. 2001.

8. J. Benaloh, M. are seeking for after, E. Horvitz, and sufficient. Lauter, "ceaseless oversaw Encryption: ensuring safety of digital medical records," Proc. ACM Workshop Cloud Computing guarantee (CCSW '09), pp. 103-114, 2009.

9. S. Yu, C. Wang, enough. Ren, and W. Lou, "undertaking cozy, Scalable,and palatable Grained records advantage admittance to manipulate in Cloud Computing," Proc. IEEE INFOCOM '10, 2010.

10. V Bozovic, D Socek, R Steinwandt, and V. I. Vil-lanyi, "Multiauthority nice essentially primarily based encryption with affordable yet curious critical master" global magazine of computer range-crunching, vol. 89,pp. 3, 2012 .

11. V. Goyal, O. Pandey, A. Sahai, and B.Waters"attribute-based encryption for first-class grained get right of entry to administer of mixed facts," in court times of the 13th ACM gathering on computer and correspondences health, pp. 8998, 2006

12. Q. Liu, G. Wang, and J. Wu, "Time primarily based virtually delegate re-encryption plan for agreeable realities taking component in a cloud state of affairs," actualities Sciences .In Press, 2012.

13. D.Boneh and M. Franklin. "person based totally truely Encryption from the Weil Pairing." In Proc. of CRYPTO'01, Santa Barbara,California, americaa., 2001.

14. J.Bethencourt, A. Sahai, and B. Waters. "Ciphertext-protection trademark essentially primarily based clearly Encryption." In Proc. of SPee7, Washington,DC, america, 2007.

15. A.Sahai and B. Waters. "Cushioned distinguishing evidence primarily based genuinely Encryption." In Proc. Of EUROCRYPT ${ }^{\text {0e } 05, ~ A a r h u s, D e n m a r k, ~} 2005$. 
16. V. Goyal, O. Pandey, A. Sahai, and B. Waters. "agreeable based virtually Encryption for remarkable grained get segment to manipulate of Encrypted facts". In Proc. of $\mathrm{CCS}^{\mathrm{ee}}$ 06, Alexandria, Virginia, the us, 2006.

17. Zhen Liu and Zhenfu Cao. On E_ciently transferring the Linear backbone chiller Sharing Scheme Matrix in Ciphertext-inclusion paintings based totally absolutely Encryption. IACR Cryptology ePrint Archive, 2010:374, 2010. 